\title{
Methodological and Technological Support for the Coordination of Smart Networks of SMEs
}

\author{
Armin Lau, Heiko Matheis, and Meike Tilebein \\ DITF Denkendorf - Centre for Management Research \\ Koerschtalstrasse 26, 73770 Denkendorf, Germany \\ \{armin.lau, heiko.matheis, meike.tilebein\} aditf-mr-denkendorf.de
}

\begin{abstract}
To develop and to manufacture highly innovative, knowledgeintensive products, processes and services, small and medium-sized enterprises (SMEs) often rely on the help of partners to be able to apply new technologies, to open up market opportunities or to extend production capabilities. Therefore, collaboration in dynamic networks has become a major success factor for SMEs. However, in many cases, companies are lacking the appropriate resources and capabilities to effectively and efficiently coordinate such collaborative activities. This paper presents a set of adaptable and easy-to-use methods and tools that support SMEs in building up, operating and terminating knowledge-driven, ICT-enabled and organizationally embedded collaboration in Smart Networks. The effectiveness will be illustrated with examples from three SME networks in different industry sectors that have applied these methods and tools in their industrial practice.
\end{abstract}

Keywords: Smart networks, network coordination, knowledge orientation, method support, tools.

\section{Collaboration Need and Collaboration Support}

Shortening product life-cycles, growing complexity and increasing market dynamics have become major challenges for companies, in particular for enterprises dealing with knowledge-intensive products and services. In order to anticipate trends and changes in the market, and in order to benefit from these rather than to lag behind requires extensive knowledge and competences in a large variety of fields. Small and medium-sized enterprises are rarely in the position to hold available such a huge knowledge base given their limited resources. They rather have to focus on core competences to stay competitive in their respective fields.

For that reason, since many years, SMEs have engaged in collaborations with other organizations to combine competences and resources and to share financial load and risks. Within the conceptual framework of collaborative networks [1], SMEs can collaborate with partners by setting up dynamic networks based on given requirements or identified opportunities.

However, industrial practice shows that operating such networks is done more on impulse than systematically. A shortage of SME-suitable methods and tools also leads 
to a strong impact of recognized weaknesses of dynamic networks: the lack of appropriate information, authority and control structures [2].

To improve the effectiveness and efficiency of such collaboration, and to at least partly overcome the above-mentioned deficits, this paper presents a set of adaptable and easy-to-use methods and tools that support SMEs in building up, operating and terminating dynamic networks. Particular attention will be given to an evenly distributed consideration of organizational, ICT and knowledge aspects of the collaboration, as promoted by the paradigm of the Smart Organization [3]. That means that methods and tools supporting Smart Networks draw equally on virtual teaming and ICT-enabled information exchange to create a knowledge-aware and knowledge-promoting collaborative environment.

\section{Industrial Model of Smart Networks}

Within the European research project SmartNets $^{1}$, a holistic industrial model for cross-sectoral SME collaboration both in development and production of knowledgeintensive products and services has been developed and evaluated in practice.

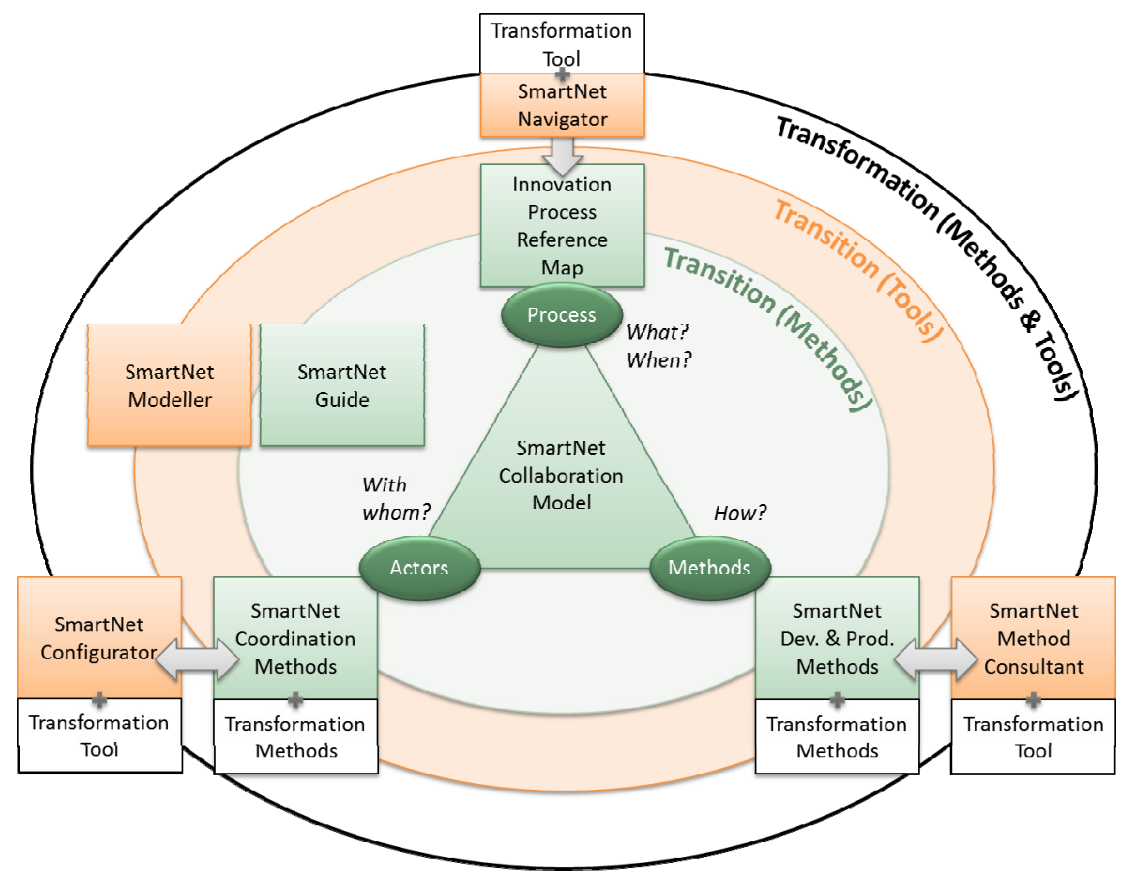

Fig. 1. Overview on SmartNets results [4]

\footnotetext{
${ }^{1}$ SmartNets, Project reference no. 262802, Website: www.smart-nets.eu
} 
The industrial model is based on the SmartNet Collaboration Model which describes collaborative innovation using the elements process, methods, actors, and their respective interrelations [5]. Numerous methods and tools support the management of these elements (see Fig. 1).

As an example, the Innovation Process Reference Map provides in-depth methodological guidance on the process and how to implement it in practice. The SmartNet Navigator implements the methodological foundation on a collaboration platform and thus offers a directly executable tool for process monitoring and execution support. One particular focus of the project was on the efficient transformation from collaborative development to production networks, thus the respective transformation results affect all three elements.

The results of SmartNets can be applied both independent of each other and in combination to answer pressing key issues of collaborative development and production [6]. In the following, this paper is going to focus on results related in particular to the actors in such collaboration, the SmartNet Modeller, the SmartNet Coordination Methodology, and the SmartNet Configurator.

\section{Method and Tool Support for Network Coordination}

For the coordination of actors in the development and production process, easily applicable methods and tools are required to provide help in the day-to-day business of SMEs. They should offer support throughout the network life-cycle, from building up to termination, and should preferably also cover all functionalities attributed to network management, namely to tasks of selection, allocation, regulation, and evaluation [7].

All four tasks are covered by the three interrelated, dedicated methods and tools that will be introduced in the following chapter. The SmartNet Modeller supports the analysis of the network as a whole and promotes a common understanding of the partnership. Based on this knowledge, systematically analyzing the risks of collaboration with the Networking Failure Mode and Effects Analysis (NFMEA) becomes much more effective. Appropriate ICT support to counter some common risks and to improve the efficiency of collaboration has been implemented on a hybrid wiki system as SmartNet Configurator. Practical case studies on the application of these results in industrial networks will follow in chapter 4.

\subsection{Modelling of Smart Networks}

One key prerequisite for acting in collaborative networks is an in-depth understanding of the network and its ecosystem. Without basic knowledge about organizational structures and responsibilities, processes, resources, knowledge, and ICT systems, tasks like the selection of appropriate partners, allocation of resources, regulation of activities, and evaluation of network performance are nearly impossible to perform in an efficient manner. This concerns many SMEs which often engage in collaboration with only limited knowledge about the network itself. 
Common approaches to business process modelling focus mainly on organizational aspects, like processes and resources. However, for knowledge-oriented networks, it is essential to understand how knowledge can be conjointly explored, retained and exploited [8]. Therefore, the approach of the Smart Network Modelling [9] focuses equally on organizational, ICT and knowledge aspects, by connecting five model types (see Fig. 2) which are recommended to be modelled top-down starting with the ecosystem. Using this modelling language enables SMEs to identify and to communicate within the network for example how conjoint knowledge creation relates to the described processes or how knowledge distribution can be supported by available IT systems.

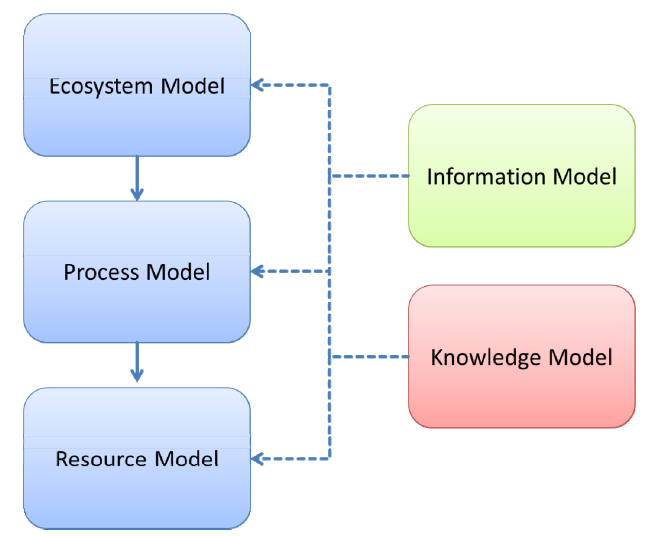

Fig. 2. Structure of Smart Network Modelling method [9]

\subsection{Networking Failure Mode and Effects Analysis (NFMEA)}

As mentioned above, sharing risks may be one motivation for a company to engage itself in collaborative innovation, but risks in collaborating are often pointed out as major reasons for companies not to collaborate with others as well. For a successful work in networks, it is the awareness of risks and a conscious, systematic handling that really can improve collaboration [10].

In terms of network management, systematically analyzing networking risks can be considered as part of the evaluation task. However, findings from this evaluation may significantly affect the other management tasks as well. For example, identifying a risk of knowledge drain will certainly affect the selection of partners, the allocation of (knowledge) resources and the contractual regulations.

One part of the SmartNet Network Coordination Methodology focuses on risks that are incurred by networking as such. For such considerations, a thorough modelling of the network, for example with the SmartNet Modeller, is a great starting point. To provide SMEs with an instrument to identify and analyze risks and to conjointly develop and implement countermeasures, a method has been developed that is based on the risk management method Failure Mode and Effects Analysis (FMEA). 
The Networking Failure Mode and Effects Analysis (NFMEA) provides a framework in which first of all each network partner on its own carries out a thorough analysis of perceived networking risks [11]. A checklist with exemplary risks from six different categories can be consulted as basis for this step. Each risk will be evaluated regarding its likelihood and severity and most critical risks are passed on into a mediated discussion amongst all network partners. Within a workshop, the identified risks from all partners will be discussed from different perspectives and will then be re-assessed. All partners decide together which of the risks have to be mitigated, and how best effects can be achieved and by whom. From this point on, a process of periodical re-evaluation and contingency planning can be initiated [11].

\subsection{Network Coordination Aspects on a Hybrid Wiki Collaboration Platform}

Within SmartNets, the hybrid wiki collaboration platform Tricia has been used for the implementation of the methodology in directly executable, easy-to-use tools. Accordingly, also support for the network coordination is provided on this hybrid wiki platform by the SmartNet Configurator.

A hybrid wiki integrates social components and features for handling of unstructured information from common wiki systems with features of structured information handling like attribute definitions and advanced querying, as they are known from databases or business intelligence software [12]. It has been chosen for the project as appropriate tool to accompany the transformation from unstructured to structured knowledge which is characteristic for the transformation from development to production.

In terms of network coordination, these hybrid structures are used to automatically evaluate regular project documentation (meeting minutes, task descriptions, result reports) in order to semantically deduce the current project status and to make recommendations and suggestions based on that. Again, this plays a significant role in the management task of evaluation, but has further impact on selection, allocation and regulation as well. The status assessment is done by the SmartNet Navigator [13] which gathers structured components from the project documentation and reasons the status according to a pre-specified rule set. The status is visualized by highlighting open, running, concluded, and overdue activities and phases in the Innovation Reference Process Model (see Fig. 3). The major advantage of this approach is that there are no additional efforts in documentation required, but the evaluation is done on information that is already available.

As the status assessment is accessible for the whole network, it creates transparency on the overall status, thus also fostering trust within the network, and it serves as an instrument for a conjoint strategic alignment. Moreover, it can be used in decision support and, as the visualization not only points out current issues but also indicates lack of documentation, can also emphasize the importance of appropriate conjoint documentation for the project planning, execution and control. It helps to reduce some of the most common risks in dynamic networks and implements some information and control structures that facilitate the network coordination. 
Based on the current status of the development, it is possible to give recommendations on which type of actors should be involved in the development. For example, when dealing with the IPR protection planning, patent consultants are crucial partners, but also R\&D departments from customer or supplier might be worthwhile to involve (see Fig. 3). In conjunction with the Smart Network Modelling, it is even possible to identify real organizations that might fit this description.

The SmartNet Configurator comprises also a set of executable methods that can support network coordination. Templates and step-by-step instructions are provided to apply for example a partner profiling to identify the right partner at the right time or to perform the above-mentioned Networking Failure Mode and Effects Analysis.

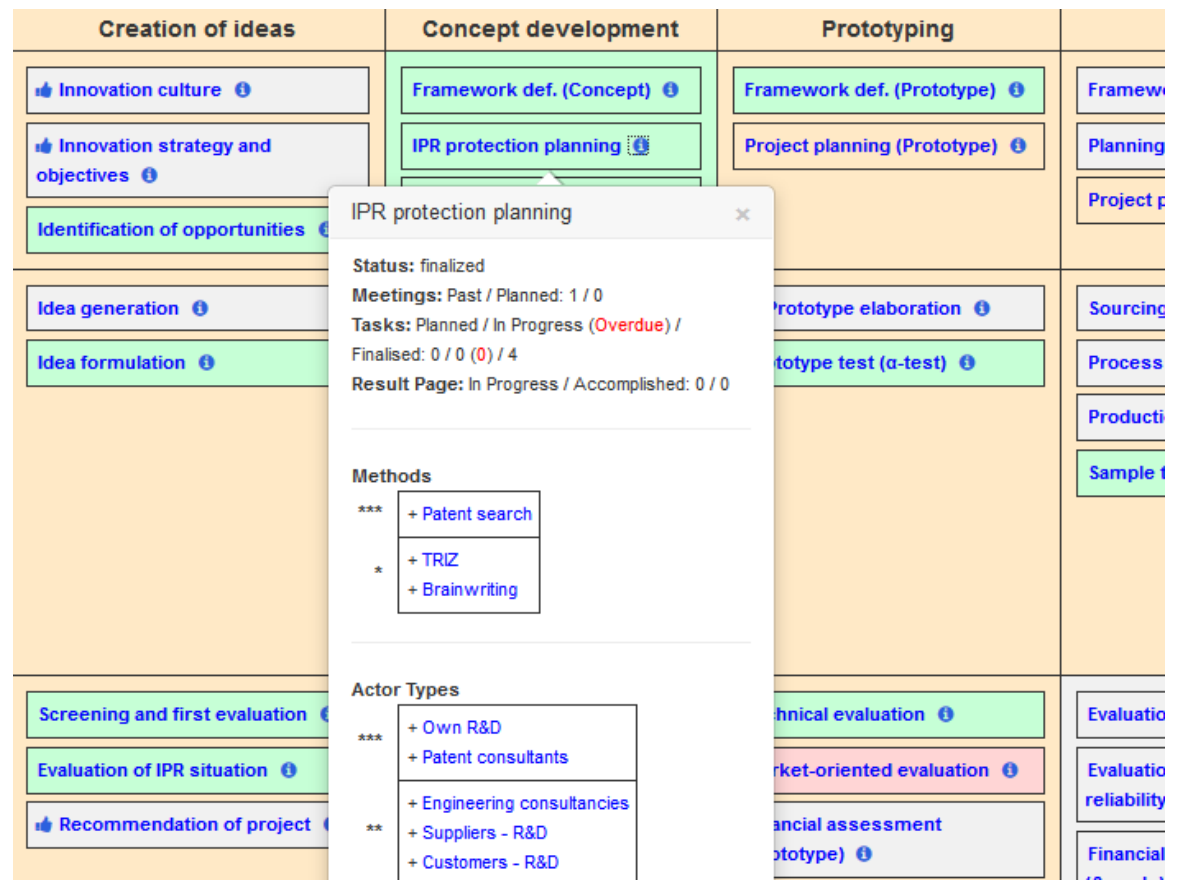

Fig. 3. Screenshot from the SmartNet Navigator, suggesting actor types for the crucial activity "IPR protection planning" [14]

\section{Use Cases}

Within SmartNets, the overall industrial model of Smart Networking and all related methods and tools have been applied in and thoroughly evaluated by three industrial SME networks. Feedback from the evaluation was directly considered in the further adaptation and refinement of the results. This also holds true for the application and assessment of the Smart Network Modeller, the Networking Failure Mode and Effects Analysis and the network coordination support on the hybrid wiki collaboration platform. All three networks have provided very positive feedback on all three elements. 
The first network consists of three SMEs and is focusing on the development on an innovative motorcycle helmet by using a high-tech material for the inner shell the helmet. At the beginning of the project, the network and its surroundings has been extensively modelled with all related processes, resources and related knowledge. While at first, the partners considered this mainly as a time consuming exercise, the resulting models proved to be very helpful in the further development. Not only were the models used to structure the conjoint documentation on the collaboration platform, they were also helping the communication between the three partners. In particular after one partner changed the responsible employee, the new co-worker could be very quickly and efficiently introduced to the project. Last but not least, after realizing the potential of the raw material, the network models were used as basis for the extension of the network into various other industry sectors [15].

In the network focusing on the development of new interior textiles, the two involved SMEs and one research organization conducted the NFMEA to assess the risks connected to networking. Even though the partners know each other for a long time, several issues were brought up during the analysis. In particular, the diverging objectives of researcher and industry partner became visible through the analysis. This led to a conjoint understanding, convincing the industry partners of the helpfulness of the method, even recommending it themselves as important for the initiation of any kind of collaboration.

Last but not least, within a network consisting of six partners who focused on the development of a medical device, the use of the hybrid wiki to support network coordination was strongly facilitating the network coordination. In this constellation with extremely long-running, interconnected development projects the tools could provide the required overview and transparency to efficiently manage contributions of all involved partners at the right time. In the final assessment, all partners agreed on the benefits provided by the tool, in particular as it strongly supported a conjoint understanding of the project and the relevance of each partner's contribution.

\section{Conclusions}

Even though the presented methods and tools are only an excerpt of the network coordination support offered by SmartNets, the practical experience from the application of these elements indicates that by offering SMEs easy-to-use, modular methods and tools for the systematic build-up, operation and termination of their networks, their effectiveness and efficiency in collaborative development will significantly improve and in the long run will also enhance their networking capabilities. An important factor is however that these methods and tools systematically promote knowledge orientation as key driver of the development.

Within SmartNets, a generalization of the overall approach has been carried out which indicates that the application of the methods and tools is not limited by industry sector or network constellation and thus can have significant impact on any manufacturing SME acting in collaborative networks. The broad applicability and the long-term effect have to be continuously evaluated in the future. 
Acknowledgments. This work has been partly funded by the European Commission through the project SmartNets: Transformation from Collaborative Knowledge Exploration Networks into Cross Sectoral and Service Oriented Integrated Value Systems (Grant Agreement No. 262806, www.smart-nets.eu). The authors wish to acknowledge the Commission for their support. We also wish to acknowledge our gratitude and appreciation to all the SmartNets Project partners for their contribution during the development of various ideas and concepts presented in this paper.

\section{References}

1. Camarinha-Matos, L.M., Afsarmanesh, H.: Collaborative networks: a new scientific discipline, Journal of Intelligent Manufacturing 16 (4), 439-452 (2005)

2. Berghoff, H., Sydow, J.: Unternehmerische Netzwerke - Theoretische Konzepte und historische Erfahrungen. In: Berghoff, H., Sydow, J. (eds.): Unternehmerische Netzwerke: Eine historische Organisationsform mit Zukunft?, pp. 9-44, Kohlhammer, Stuttgart (2007)

3. Filos, E.: Smart Organizations in the Digital Age, In: Mezgár, I. (ed.): Integration of ICT in Smart Organizations, pp. 1-37, Idea Group Publishing, Hershey (2006)

4. Lau, A., Tilebein, M., Fischer, Th.: The industrial model of Smart Networks for SME collaboration - Implementation and success stories, In: Workshop Proceedings of I-ESA 2014, 24-28 March 2014, ISTE, forthcoming.

5. Lau, A., Fischer, Th., Hirsch, M., Matheis, H.: SmartNet collaboration model-a framework for collaborative development and production, In: Katzy, B., Holzmann, T., Sailer, K., Thoben, K.-D. (eds.): Innovation by collaboration and entrepreneurial partnerships. Proceedings of the 18th International Conference on Concurrent Enterprising (ICE 2012). Munich, 18.-20.06.2012, pp. 519-528 (2012)

6. SmartNets: D9.2 SmartNet Compendium - SmartNets industrial model and guidelines for its application. Public Deliverable (2014)

7. Sydow, J.: Management von Netzwerkorganisationen - Zum Stand der Forschung, In: Sydow, J. (ed.): Management von Netzwerkorganisationen: Beiträge aus der "Managementforschung", pp. 387-472, Gabler, Wiesbaden (2006)

8. Lichtenthaler, U., Lichtenthaler, E.: A Capability-Based Framework for Open Innovation: Complementing Absorptive Capacity. Journal of Management Studies, 46(8), 1315-1338 (2009)

9. SmartNets: D1.2 SmartNets Modeller and application guidelines, Public Deliverable (2011) 10.Lau, A., Tilebein, M.: Kompetenz des Netzwerks durch Kompetenz des Netzwerkens Förderung der Netzwerkfähigkeit kleiner und mittlerer Unternehmen, In: Biedermann, H. (ed.): Corporate Capability Management - Wie wird kollektive Intelligenz im Unternehmen genutzt?, GITO, Berlin (2013)

11.Kempf, A.: Dynamische Vernetzung von Unternehmen - Entwicklung einer Methodik zum systematischen Umgang mit Vernetzungsrisiken, University of Stuttgart, Bachelor Thesis (2012)

12.Matthes, F., Neubert, C., Steinhoff, A.: Hybrid wikis: Empowering users to collaboratively structure information. In: 6th International Conference on Software and Data Technologies (ICSOFT), Sevilla (2011) 
13.Matheis, H., Lau, A., Hirsch, M.: Technological support for managing collaborative innovation projects in SME networks, In: Conference Proceedings of the IEEE Technology Management Conference \& 19th ICE Conference, The Hague, The Netherlands (2013)

14.SmartNets: D6.1 SmartNet Configurator and application guidelines, Public Deliverable (2013)

15.Paronetto, G. (2013). Protective Helmet for Motorbike Market. „Make it quickly, make it smartly, ... deliver it when it's needed" - Our Experience. Presentation on Prosumer.Net Final Conference, May 27/28 2013, Brussels (2013) 